\title{
ABOUT DIRECTED UNIONS OF ARTINIAN SUBRINGS OF A VON NEUMANN REGULAR RING
}

\author{
DRISS KARIM
}

(Received 26 February 2003; revised 31 March 2004)

Communicated by J. Du

\begin{abstract}
This work is concerned with the question of when a von Neumann regular ring is expressible as a directed union of Artinian subrings.

2000 Mathematics subject classification: primary 13A99; secondary 13A15, 13B02, $13 E 05$.

Keywords and phrases: Von Neumann regular ring, residue fields, zero-dimensional ring, semi-quasilocal ring, directed union of Artinian subrings.
\end{abstract}

\section{Introduction}

Given a commutative ring $R$, we recall that $R$ is von Neumann regular if for each element $r$ in $R$ there exists an element $r^{\prime}$ such that $r=r^{2} r^{\prime}$, or equivalently, $R$ is reduced ( 0 is the only nilpotent element) and zero-dimensional (all prime ideals are maximal). Several papers in the literature have dealt with various aspects of von Neumann regular rings in commutative ring theory (see, for example, $[5,6,8]$ ). These works are source of motivation for this paper.

The purpose of this paper is to pursue the study of the problem of whether a von Neumann regular ring $R$ is expressible as a directed union of Artinian subrings, raised by Gilmer and Heinzer in 1992 [1, Problem 42].

This fact leads us to consider the family of residue fields of $R$ denoted $\mathscr{F}(R)$. We focus on the behaviour of the family of the residue fields of $R$. It is well known that if a zero-dimensional ring $R$ with $\operatorname{Idem}(R)$ is finite, then $R$ is a directed union of Artinian subrings [6, Theorem 5.4]. This leads us to examine the case where $\operatorname{Idem}(R)$ is infinite.

(C) 2005 Australian Mathematical Society 1446-7887/05 $\$ A 2.00+0.00$ 
Let $\left\{R_{\alpha}\right\}_{\alpha \in A}$ be a nonempty family of rings and $\prod_{\alpha \in A} R_{\alpha}$ their product. We frequently consider $\prod_{\alpha \in A} R_{\alpha}$ as the set of all functions $f: A \rightarrow \bigcup_{\alpha \in A} R_{\alpha}$, such that $f(\alpha) \in R_{\alpha}$ for each $\alpha \in A$, with addition and multiplication defined pointwise: $(f+g)(\alpha)=f(\alpha)+g(\alpha)$ and $(f g)(\alpha)=f(\alpha) g(\alpha)$. In this perspective, the direct sum ideal of $\prod_{\alpha \in A} R_{\alpha}$, denoted by $\bigoplus_{\alpha \in A} R_{\alpha}$, is the set of functions $f \in \prod_{\alpha \in A} R_{\alpha}$ that are finitely nonzero (that is, $\left\{\alpha \in A: f(\alpha) \neq 0\right.$ in $\left.R_{\alpha}\right\}$ is finite).

All rings considered in this paper are assumed to be commutative with a unity element. If $S$ is a subring of a ring $R$, we assume that $R$ and $S$ have the same unity element. We denote by $\mathscr{A}(R)$ and $\mathscr{C}(R)$, respectively, the set of Artinian subrings of $R$, and the set $\{\operatorname{char}(R / M): M$ is a maximal ideal of $R\}$.

\section{Directed unions of Artinian subrings}

Let us fix notation for much of Section 2. The data will consist of a directed system $\left(R_{j}, f_{j k}\right)$ of rings indexed by a directed set $(I, \leq)$. Let $R=\bigcup_{j \in I} R_{j}$, together with the canonical maps $f_{j}: R_{j} \rightarrow R$. The ring $R$ is said to be a directed union of the $R_{j}$ 's if the $f_{j k}$ 's are inclusion maps. Thus, directed unions can be treated by assuming all $f_{j k}$ to be monomorphisms. Notice that if $R_{j}$ is a ring for each $j \in I$, then $R$ is also a ring. However, $R$ needs not be Artinian even if each $R_{j}$ is. If $R=\bigcup_{j \in I} R_{j}$ is a directed union of Artinian subrings, then we regard each $R_{i}$ as a subrings of $R$, that is, contains the same unity. Turning to the Artinian case, two key properties of an Artinian ring $R$ that come into play are that $\operatorname{Spec}(R)$ is finite and that $R$ has only finitely many idempotents.

We begin this section with two lemmas that we use throughout this paper.

LEMMA 2.1. Let $R$ be a ring.

(1) (a) If $R$ is a directed union of Artinian subrings then $\mathscr{C}(R)$ is finite.

(b) If $\mathscr{C}(R)$ is infinite then $\mathscr{A}(R)=\emptyset$.

(2) If $R$ is a zero-dimensional ring with finite spectrum, then $R$ is expressible as a finite product of zero-dimensional quasi-local subrings.

(3) If $R$ is a von Neumann regular ring, then $R$ is Artinian if and only if $R$ is a finite product of fields if and only if $R$ is Noetherian.

PROOF. (1) (a) Suppose that $R$ is a directed union of Artinian subrings. Then $\mathscr{A}(R) \neq \emptyset$ and hence $\mathscr{C}(R)$ is finite [4, Proposition 1].

(b) Assume that $\mathscr{A}(R) \neq \emptyset$, let $S \in \mathscr{A}(R)$, then $\mathscr{C}(R) \subseteq \mathscr{C}(S)$ and hence $\mathscr{C}(S)$ is infinite, a contradiction with $S$ is Artinian ring ( $\mathscr{C}(R)$ is finite).

(2) Let $\operatorname{Spec}(R)=\left\{M_{i}\right\}_{i=1}^{n}$ be the spectrum of $R$. Let $S_{M_{i}}(0)$ denote $\operatorname{Ker} \varphi_{i}$ for each $i=1, \ldots, n$, where $\varphi_{i}: R \rightarrow R_{M_{i}}$ and $\varphi_{i}(r)=r / 1$, is the canonical homomorphism. 
Since $\operatorname{Rad}\left(S_{M_{i}}(0)\right)=M_{i}, S_{M_{i}}(0)$ is a primary ideal. Note that $\bigcap_{i=1}^{n} S_{M_{i}}(0)=(0)$ and $S_{M_{i}}(0)+S_{M_{j}}(0)=R$ for each $i \neq j$ in $\{1, \ldots, n\}$. Therefore, $R \simeq R / \bigcap_{i=1}^{n} S_{M_{i}}(0)$. By the Chinese Remainder Theorem, $R \simeq \prod_{i=1}^{n} R / S_{M_{i}}(0)$, where $R / S_{M_{i}}(0)$ is quasilocal and zero-dimensional, for $i=1, \ldots, n$.

(3) This follows from the fact that a von Neumann regular ring with only finitely many idempotent elements is a finite product of fields, and then it is Artinian.

Lemma 2.1 leads us to state that if $R$ is a von Neumann regular ring, then $R$ is a directed union of Artinian subrings if and only if $R$ is a directed union of zerodimensional semi-quasilocal subrings. This follows from the fact that a von Neumann regular ring with only finitely many idempotent elements is a finite product of fields and then it is Artinian.

LEMMA 2.2. Let $R$ be a ring and $S$ a multiplicatively closet subset of $R$. Then:

(a) If $R$ is a directed union of Artinian subrings, then so is $S^{-1} R$.

(b) $R$ is a directed union of zero-dimensional semi-quasilocal subrings if and only if $S^{-1} R$ is a directed union of zero-dimensional quasilocal subrings.

(c) If $R$ is reduced, then $R$ is a directed union of Artinian subrings if and only if $S^{-1} R$ is a directed union of Artinian subrings.

PROOF. (a) If $R=\bigcup_{i \in I} R_{i}$ is a directed union of Artinian subrings, then $S^{-1} R=$ $\bigcup_{i \in I} S_{i}^{-1} R_{i}$, where $S_{i}=S \cap R_{i}$ for each $i \in I$. By [11, (6.17)], $S_{i}^{-1} R_{i}$ is Noetherian for each $i \in I$ since each $R_{i}$ is Noetherian. Each $S^{-1} R_{i}$ is zero-dimensional as each $R_{i}$ is (see, for example, [2, Proposition 1.21]). By [3, Theorem 8.5], $S^{-1} R_{i}$ is an Artinian ring for each $i \in I$. The family $\left\{S^{-1} R_{i}\right\}_{i \in I}$ is directed because so is the family $\left\{R_{i}\right\}_{i \in I}$. Thus $S^{-1} R$ is a directed union of Artinian subrings.

(b) Suppose that $R=\bigcup_{i \in I} R_{i}$ is a directed union of zero-dimensional semiquasilocal subrings, we have in (a) $S^{-1} R=\bigcup_{i \in l} S_{i}^{-1} R_{i}$. Let $\operatorname{Spec}\left(S^{-1} R\right)=\left\{S^{-1} \mathrm{~m}\right.$ : $\mathfrak{m} \in \operatorname{Spec}(R)$ and $\mathfrak{m} \cap S=\emptyset$, then $S_{i}^{-1} R_{i}$ is semi-quasilocal. From (a) we conclude that $S^{-1} R$ is a directed union of the required type. Conversely, suppose that $S^{-1} R=\bigcup_{i \in I} T_{i}$ is a directed union of zero-dimensional semi-quasilocal subrings. Let $R_{i}=T_{i} \cap R$, then $R_{i}$ is zero-dimensional (see, for example, [7, Theorem 2.4]). Also $\left|\operatorname{Idem}\left(R_{i}\right)\right| \leq\left|\operatorname{Idem}\left(T_{i}\right)\right|$. Any zero-dimensional ring with only finitely many idempotent elements is a semi-quasilocal ring. Therefore, $R=\bigcup_{i \in I} R_{i}$ is a directed union of zero-dimensional semi-quasilocal subrings.

(c) First, we claim that if $R$ is reduced, then so is $S^{-1} R$. Let $r / s \in N\left(S^{-1} R\right)$, where $N\left(S^{-1} R\right)$ is the nilradical of $S^{-1} R$. Then there exists $n_{o} \in \mathbb{N}^{*}$ such that $(r / s)^{n_{o}}=0$; so there exists $u \in U$ such that $(r u)^{n_{o}}=0$. Since $R$ is reduced, we have $r u=0$ and hence $r / s=0$. In other words, $N\left(S^{-1} R\right)=(0)$. Thus, $S^{-1} R$ is reduced. Now, assume that $S^{-1} R=\bigcup_{i \in I} T_{i}$ is a directed union of Artinian subrings. By [10, Corollary 4], $R$ is also a directed union of Artinian subrings. 
EXAMPLE 2.3. Let $R=\mathbb{Q}^{\omega_{o}}$ be a countable direct product of copies of $\mathbb{Q}$, where $\mathbb{Q}$ denotes the field of rational numbers. We consider

$$
\mathbb{Q}^{(i)}=\left\{\left\{x_{j}\right\}_{j=1}^{\infty} \in \mathbb{Q}^{w_{o}}: x_{i-1}=x_{i}=\cdots\right\}
$$

a subring of $R$, and $\mathbb{Q}^{(i)} \simeq \mathbb{Q}^{i}$, the product of $i$ copies of $\mathbb{Q}$, an Artinian von Neumann regular ring. It follows that $\mathscr{A}(R) \neq \emptyset$. We can easily see that $\mathbb{Q}^{(i)}, i \geq 1$, are the only Artinian subrings of $R$. However, $R$ is not a directed union of Artimian subrings. Indeed, consider $y=\left(y_{i}\right)_{i \in \mathbb{N}^{*}} \in R$ such that $y_{i} \neq y_{j}$ for $i \neq j$. Then, for each $i \in \mathbb{N}^{*}$, $y \notin \mathbb{Q}^{(i)}$. It follows that $\lim \mathbb{Q}^{(i)} \varsubsetneqq \mathbb{Q}^{\omega_{o}}$. However $\mathscr{S}=\lim \mathbb{Q}^{(i)}$ is the biggest subring of $R$ which expressible as a directed union of Artinian $\overrightarrow{\text { subrings. }}$

Hence $\mathscr{A}(R) \neq \emptyset$ does not imply that $R$ is a directed union of Artinian subrings.

Let $R$ be a von Neumann regular ring and $\left\{M_{\lambda}\right\}_{\lambda \in \Lambda}$ its spectrum. Since $R$ is a reduced ring, we have $\bigcap_{\lambda \in \Lambda} M_{\lambda}=(0)$. This allows us to regard $R$ as a subring of $S=\prod_{\lambda \in \Lambda} R / M_{\lambda}$. It is known that if there exists $k \in \mathbb{Z} \backslash\{0\}$ such that the set $\left\{\lambda \in \Lambda:\left|R / M_{\lambda}\right|>k\right\}$ is finite, then $\prod_{\lambda \in \Lambda} R / M_{\lambda}$ is a directed union of Artinian subrings [6, Theorem 6.7]. By [10, Corollary 4], $R$ is also a directed union of Artinian subrings. Hence, we further assume that for each $k \in \mathbb{Z}^{*}$ the set $\{\lambda \in \Lambda$ : $\left.\left|R / M_{\lambda}\right|>k\right\}$ is infinite. Under these assumptions, it would be interesting to know whether $\mathscr{A}\left(R, \prod_{\lambda \in \Lambda} R / M_{\lambda}\right) \neq \emptyset$, where $\mathscr{A}\left(R, \prod_{\lambda \in \Lambda} R / M_{\lambda}\right)$ is the family of Artinian subrings of $S$ containing $R$. The following result provides an answer to this problem.

PROPOSITION 2.4. Let $R$ be a von Neumann regular ring with $\operatorname{Spec}(R)=\left\{M_{\lambda}\right.$ : $\lambda \in \Lambda\}$. The following statements are equivalent:

(i) $\mathscr{A}\left(R, \prod_{\lambda \in \Lambda} R / M_{\lambda}\right) \neq \emptyset$.

(ii) There exist $\lambda_{1}, \ldots, \lambda_{n}$ in $\Lambda$ such that $\bigcap_{i=1}^{n} M_{\lambda_{i}}=(0)$.

(iii) $\operatorname{Idem}(R)$ is a finite set.

(iv) $R$ is Artinian.

PROOF. (i) $\Rightarrow$ (ii). If $\mathscr{A}\left(R, \prod_{\lambda \in \Lambda} R / M_{\lambda}\right) \neq \emptyset$, then there exists an Artinian ring $A$ such that $R \subseteq A \subset \prod_{\lambda \in \Lambda} R / M_{\lambda}$. Since $\prod_{\lambda \in \Lambda} R / M_{\lambda}$ is reduced, so is the ring A. Therefore, $A \simeq \prod_{i=1}^{n} K_{i}$, where $K_{i}$ is a field for each $i=1, \ldots, n$. Now, let $Q_{i}=\prod\left\{K_{j}: j=1, \ldots, n\right.$ and $\left.j \neq i\right\}$, where $i=1, \ldots, n$. Then $\prod_{j=1}^{n} K_{j} / Q_{i} \simeq K_{i}$ and hence $Q_{i} \in \operatorname{Spec}(A)$. Thus, for each $i=1, \ldots, n, M_{\lambda_{i}}=Q_{i} \cap R \in \operatorname{Spec}(R)$ and $\bigcap_{i=1}^{n} M_{\lambda_{1}}=(0)$.

(ii) $\Rightarrow$ (iii). Since $\bigcap_{i=1}^{n} M_{\lambda_{i}}=(0)$, then $R$ is imbeddable in $\prod_{i=1}^{n} R / M_{\lambda_{i}}$. Hence $R$ has only finitely many idempotent elements.

(iii) $\Rightarrow$ (iv). It follows form [10, Lemma 1].

(iv) $\Rightarrow$ (i). Obvious. 
Let $S \subseteq T$ be an extension of rings with $S$ an Artinian and von Neumann regular, and let $F$ be a finite set of idempotents of $T$ then $S[F]$ is Artinian. Indeed, $S[F]$ is an integral extension of $S$ since each idempotent element $e$ of $F$ satisfies $e^{2}=e$. Therefore, $\operatorname{dim} S[F]=0$. Furthermore, $S[F]$ is finitely generated over the Noetherian ring $S$. Hence $S[F]$ is Noetherian and then Artinian.

LEMMA 2.5. Let $R$ be $a$ von Neumann regular subring of a ring $T$ and $\operatorname{Idem}(T)=$ $\left\{e_{\lambda}\right\}_{\lambda \in \Lambda}$ the set of idempotent elements of $T$. Then $R$ is a directed union of Artinian subrings if and only if $R\left[\left\{e_{\lambda}: \lambda \in \Lambda\right\}\right]$ is a directed union of Artinian subrings.

PROOF. Assume that $R=\bigcup_{i \in I} R_{i}$ is a directed union of Artinian subrings. We write $\Lambda=\bigcup_{j \in J} \Lambda_{j}$, where $\Lambda_{j}$ ranges over all finite subsets of $\Lambda$, thus

$$
\left\{e_{\lambda}\right\}_{\lambda \in \Lambda}=\bigcup_{j \in J}\left\{e_{\lambda}\right\}_{\lambda \in \Lambda_{j}}, \quad \text { and } \quad R\left[\left\{e_{\lambda}: \lambda \in \Lambda\right\}\right]=\bigcup_{(i, j) \in I \times J} R_{i}\left[\Lambda_{j}\right],
$$

where $R_{i}\left[\Lambda_{j}\right]=R_{i}\left[\left\{e_{k}: k \in \Lambda_{j}\right\}\right]$. Now, endowed with the lexicographic order, the set $I \times J$ is directed and clearly the family $\left\{R_{i}\left[\Lambda_{j}\right]:(i, j) \in I \times J\right\}$ is also directed. It follows that $R\left[\left\{e_{\lambda}: \lambda \in \Lambda\right\}\right]$ is a directed union of Artinian subrings. The converse follows from [10, Lemma 1 and Corollary 4$]$.

PROPOSITION 2.6. Let $\left\{K_{i}\right\}_{i \in I}$ be a family of fields. Then the following statements are equivalent:

(i) $\mathscr{A}\left(\prod_{i \in I} K_{i}\right) \neq \emptyset$.

(ii) $\left\{\operatorname{char}\left(K_{i}\right): i \in I\right\}$ is finite.

ProOF. (i) $\Rightarrow$ (ii). Let $A$ be an Artinian subring of $\prod_{i \in I} K_{i}$. By [4, Proposition 1] $\mathscr{C}\left(\prod_{i \in I} K_{i}\right) \subseteq \mathscr{C}(A)$. Since $A$ is Artinian, $\operatorname{Max}(A)$ is finite and hence $\mathscr{C}(A)$ is finite. Thus $\left\{\operatorname{char}\left(K_{i}\right): i \in I\right\}$ is finite since $\left\{\operatorname{char}\left(K_{i}\right): i \in I\right\} \subseteq \mathscr{C}\left(\prod_{i \in I} K_{i}\right)$.

(ii) $\Rightarrow$ (i). If $\operatorname{char}\left(\prod_{i \in I} K_{i}\right) \neq 0$, then $\mathscr{A}\left(\prod_{i \in I} K_{i}\right) \neq \emptyset$, since the prime subring of $\prod_{i \in I} K_{i}$ is finite, and hence it is Artinian. So suppose that char $\left(\prod_{i \in I} K_{i}\right)=0$. Since $\left\{\operatorname{char}\left(K_{i}\right): i \in I\right\}=\left\{p_{1}, \ldots, p_{k}\right\}$ is finite, we may set $I=A_{1} \cup \cdots \cup A_{k}$, where $A_{s}=\left\{i \in A: \operatorname{char}\left(K_{i}\right)=p_{s}\right\}$ for $s=1, \ldots, k$. Since $\operatorname{char}(R)=0$, we may suppose that $p_{1}=0$ and write $R=R_{1} \times \cdots \times R_{k}$, where $R_{i}=\prod_{j \in A_{i}} K_{j}$, for each $i=1, \ldots, k$. Hence $\mathbb{Q}^{*} \times \pi_{2}^{*} \times \cdots \times \pi_{k}^{*} \in \mathscr{A}(R)$, where $\pi_{i}$ is the prime subring of $R_{i}$ for each $i=2, \ldots, k$ and $\mathbb{Q}^{*}$ (respectively, $\pi_{i}^{*}$ ) denotes the diagonal imbedding of $\mathbb{Q}$ (respectively, $\pi_{i}$ ) into $\prod_{j \in A_{1}} K_{j}$ (respectively, $\prod_{j \in A_{i}} K_{j}$, for $i=2, \ldots, k$ ).

Let $\mathscr{B}\left(R, \prod_{\alpha \in A} R / M_{\alpha}\right)$ denote the set of all the intermediate directed unions of Artinian subrings between $R$ and $\prod_{\alpha \in A} R / M_{\alpha}$. By Example 2.3, it may happen that $\mathscr{B}\left(R, \prod_{\alpha \in A} R / M_{\alpha}\right) \neq \emptyset$ even if $\prod_{\alpha \in A} R / M_{\alpha}$ is not a directed union of Artinian 
subrings. In this case $R$ must be a directed union of Artinian subrings. By [5, Corollary 1.2], if $\mathscr{C}(R)$ is infinite, then $R$ admits no Artinian subring. So we restrict our study to the case when $\mathscr{C}(R)$ is a finite set.

If $x \in N(R)$, we denote by $\eta(x)$ the index of nilpotency of $x$, that is, $\eta(x)=k$ if $x^{k}=0$ but $x^{k-1} \neq 0$. We define $\eta(R)$ to be $\operatorname{Sup}\{\eta(x): x \in N(R)\}$; if the set $\{\eta(x)$ : $x \in N(R)\}$ is unbounded, then we write $\eta(R)=\infty$. From [6, Theorem 3.4], we have $\operatorname{dim} \prod_{\alpha \in A} T_{\alpha}=0$, for any family $\left\{T_{\alpha}\right\}_{\alpha \in A}$ of zero-dimensional rings, equivalent to $\left\{\alpha \in A: \eta\left(T_{\alpha}\right)>k\right\}$ is finite for some $k \in \mathbb{Z}^{+}$.

It is worth to mention that $\prod_{\alpha \in A} R_{\alpha}$ needs not be a directed union of Artinian subrings even if $\operatorname{dim}\left(\prod_{\alpha \in A} R_{\alpha}\right)=0$, where $\left\{R_{\alpha}\right\}_{\alpha \in A}$ is family of zero-dimensional rings. For instance, let $K$ be an infinite field, $X$ be an indeterminate over $K$, and $R_{i}=K[X] /\left(X^{3}\right)$ for each $i \in \mathbb{Z}^{+}$. We have $N\left(R_{i}\right)=(X) /\left(X^{3}\right), i \in \mathbb{Z}^{+}$. Since $\eta\left(R_{i}\right)=3$ for each $i \in \mathbb{Z}^{+}$, by [6, Theorem 3.4], $\operatorname{dim} \prod_{i=1}^{\infty} R_{i}=0$ and from [6, Theorem 6.7], $\prod_{i=1}^{\infty} R_{i}$ is not a directed union of Artinian subrings.

Let $R$ be a von Neumann regular ring and $\operatorname{Max}(R)=\left\{M_{\lambda}\right\}_{\lambda \in A}$ the set of maximal ideals of $R$. Let $\varphi: R \hookrightarrow \prod_{\lambda \in A} L_{\lambda}$ be a monomorphism defined by $\varphi(x)=\left\{x_{\lambda}\right\}_{\lambda \in A}$ such that $x_{\lambda} \equiv \bar{x}$ modulo $M_{\lambda}$, where $R / M_{\lambda} \simeq L_{\lambda}$ for each $\lambda \in A$. We assume that $\mathscr{C}(R)=\left\{p_{1}, \ldots, p_{n}\right\}$ is finite, then we have $\prod_{\lambda \in A} L_{\lambda}=\bigoplus_{j=1}^{n} R_{j}$, where $R_{j}=$ $\prod_{\lambda \in A_{j}} L_{\lambda}$ and $A_{j}=\left\{\lambda \in A: \operatorname{char}\left(L_{\lambda}\right)=p_{j}\right\}$. For each $j$, we suppose there exists a field $\Omega_{j}$ that contains all fields $L_{\lambda}, \lambda \in A_{j}$. We now regard $R$ as a subring of $\prod_{j=1}^{n} S_{j}$, where $S_{j}=\Omega_{j}^{l_{j}}$. Let $e_{j}$ be the primitive idempotent of $\prod_{j=1}^{n} S_{j}$ associated with $\{j\}$ for $j=1, \ldots, n$. Then $R\left[e_{1}, \ldots, e_{n}\right]=R e_{1} \oplus \cdots \oplus R e_{n}$. Therefore, $R$ is a directed union of Artinian subrings if and only if $R\left[e_{1}, \ldots, e_{n}\right]$ is a directed union of Artinian subrings if and only if every $R e_{i}$ is a directed union of Artinian subrings. Also $R e_{j} \subseteq \Omega_{j}^{l_{j}}$. We denote by $\Omega_{j}^{*}$ the diagonal imbedding of $\Omega_{j}$ into $\Omega_{j}^{l_{j}}$. Without loss of generality we assume that $R e_{j}=R$, In other words, $\mathscr{C}(R)=\{p\}$.

THEOREM 2.7. Let $R$ be a von Neumann regular ring with $\mathscr{F}(R)=\left\{L_{\alpha}\right\}_{\alpha \in A}$, $\mathscr{C}(R)=\{p\}$, and $\mathscr{S}=\left\{\left\{r_{\alpha}\right\}_{\alpha \in A} \in \prod_{\alpha \in A} L_{\alpha}:\left\{r_{\alpha}\right\}_{\alpha \in A}\right.$ has only finitely many distinct coordinates $\}$. Assume that each $L_{\alpha}$ is absolutely algebraic and there exists a field $\Omega$ that contains all but finitely many $L_{\alpha}$ 's. Then $\mathscr{S}$ is a directed union of Artinian subrings.

PROOF. To show that $\mathscr{S}$ is a directed union of Artinian subrings, it suffices to prove that $\mathscr{S}$ is covered by a directed union of finite products of fields. Let $f \in \mathscr{S}$, then $\{f(\alpha): \alpha \in A\}=\left\{f_{1}, \ldots, f_{t}\right\}$ a finite set. Let $A_{i}=\left\{\alpha \in A: f(\alpha)=f_{i}\right\}$ and denote $f_{i}^{*}=\left(f_{i}, f_{i}, \ldots, f_{i}, \ldots\right) \in \prod_{\alpha \in A_{i}} L_{\alpha}$. Then $\{f(\alpha)\}_{\alpha \in A}=\left(f_{1}^{*}, \ldots, f_{i}^{*}\right)$. Since $A=\bigcup_{i=1}^{t} A_{i}$, and all the fields $L_{\alpha}, \alpha \in A_{i}$, have the same characteristic, then, up to isomorphism, $\bigcap_{\alpha \in A_{i}} L_{\alpha}=K_{i}$ is a field with $f_{i}^{*} \in K_{i}^{*}$, the diagonal imbedding of $K_{i}$ in $\prod_{\alpha \in A_{i}} L_{\alpha}$. It follows that $f \in K_{1}^{*} \times \cdots \times K_{t}^{*} \simeq K_{1} \times \cdots \times K_{t}$. Therefore, 
$\mathscr{S}$ is covered by a directed union of Artinian subrings.

EXAMPLE 2.8. Let $p$ be a positive prime integer and $\left\{q_{i}\right\}_{i \in \mathbb{N}^{*}}$ be an infinite family of distinct prime integers. Let $\mathscr{F}=\{\mathbb{Q}\} \cup\left\{\mathbb{Q}\left(\zeta_{i}\right)\right\}_{i=1}^{\infty}$ be an infinite family of fields, where $\zeta_{i}$ is a $p^{q_{i}}$-primitive root of unity. We denote by $\varphi_{i}$ the imbedding of $\mathbb{Q}$ into $\mathbb{Q}\left(\zeta_{i}\right)$ for each $i \in \mathbb{Z}^{+}$. Let $\varphi=\prod_{i=1}^{\infty} \varphi_{i}, T=\prod_{i=1}^{\infty} \mathbb{Q}\left(\zeta_{i}\right)$ and let $I=\bigoplus_{i=1}^{\infty} \mathbb{Q}\left(\zeta_{i}\right)$ be the direct sum ideal of $T$. We denote by $\mathbb{Q}^{*}=\varphi(\mathbb{Q})=R_{o} \simeq \mathbb{Q}$ the diagonal imbedding of $\mathbb{Q}$ in $T$. Let $S=R_{o}+I$, since $S$ is a subring of $T$ and $\operatorname{dim}(S)=0[9$, Proposition 2.7], the ring $S$ is a Von Neumann regular ring. Let

$$
\begin{aligned}
& S_{1}=\mathbb{Q}\left(\zeta_{1}\right) \times \mathbb{Q}^{*} \simeq \mathbb{Q}\left(\zeta_{1}\right) \times \mathbb{Q}, \quad \cdots, \\
& S_{i}=\mathbb{Q}\left(\zeta_{1}\right) \times \cdots \times \mathbb{Q}\left(\zeta_{i}\right) \times \mathbb{Q}^{*} \simeq \mathbb{Q}\left(\zeta_{1}\right) \times \cdots \times \mathbb{Q}\left(\zeta_{i}\right) \times \mathbb{Q} .
\end{aligned}
$$

We have that $S_{j} \subset S_{j+1}$, for each positive integer $j \in \mathbb{Z}^{+}$. Therefore, $S=\bigcup_{i \in \mathbb{N}^{*}} S_{i}$ is an increasing union of Artinian subrings.

THEOREM 2.9. Let $R$ be a von Neumann regular ring and $\mathscr{F}(R)=\left\{L_{\alpha}\right\}_{\alpha \in A}$ the set of residue fields of $R$. Suppose that each $L_{\alpha}$ is absolutely algebraic. Then $R$ is a directed union of Artinian subrings if and only if for each $x=\left\{x_{\lambda}\right\}_{\lambda \in A} \in R, x$ has only finitely many distinct coordinates.

PROOF. Assume that $R=\bigcup_{i \in I} R_{i}$ is a directed union of Artinian subrings. Since $R$ is imbeddable in $\prod_{\lambda \in A} R / M_{\lambda}$, we regard $R$ as a subring of $\prod_{\lambda \in A} R / M_{\lambda}$, and hence each $R_{i}$ is a subring of $\prod_{\lambda \in A} R / M_{\lambda}$. Let $x=\left\{x_{\lambda}\right\}_{\lambda \in A} \in R$ then $x \in R_{i_{o}}$ for some $i_{o} \in I$. Since each $R_{i}$ is isomorphic to a finite product of fields, say $\prod_{i=1}^{n} K_{i}$, which in turn is isomorphic to $\simeq \prod_{i=1}^{n} K_{j}^{*}$, where $K_{j}^{*}$ is the diagonal imbedding of $K_{i}$ into $\prod_{i \in A_{i}} R / M_{\lambda}$ and $A_{i}=\left\{\lambda \in A: x_{\lambda}=x_{i}\right\}$. It follows that $\left\{x_{\lambda}\right\}_{\lambda \in A}=\left(x_{1}^{*}, \ldots, x_{n}^{*}\right)$ with $x_{i} \in K_{i}, i \in\{1, \ldots, n\}$. The converse follows from the fact that the $R \subseteq \mathscr{S}$ (see Theorem 2.7 and [ 10 , Corollary 4$])$.

EXAMPLE 2.10. Let $F$ be an absolutely algebraic infinite field, $\alpha$ an infinite cardinal, and $S$ the direct product of $\alpha$ copies of $F$. Let $A$ be a set of cardinality $\alpha$ and consider $S$ as the set of all functions $f: A \rightarrow F$ under pointwise addition and multiplication. Let $R=\{f \in S: f(A)$ is finite $\}$, let $\mathscr{P}=\left\{A_{1}, \ldots, A_{n}\right\}$ be any finite partition of $A$, where $A_{i}$ is a subset of $A$ for each $i$. Moreover, $R_{\mathscr{P}}=\{f \in S: f$ is constant on each $\left.A_{i}\right\}$ is a subring of $R$ containing $\pi$, the prime subring of $S$. Clearly, $R_{\mathscr{P}} \simeq F^{n}$. We use $F^{*}$ to denote the diagonal imbedding of $F$ in $S$. From the proof of [6, Proposition 5.2], the family $\left\{R_{\mathscr{P}}: \mathscr{P}\right.$ is a finite partition of $\left.A\right\}$ is directed. Each $R_{\mathscr{P}}$ is Artinian and von Neumann regular and $R=\cup R_{\mathscr{S}} \simeq F^{*}+I$, where $I=\bigoplus_{\beta \in A} F$ is the direct sum ideal of $S$, is the biggest subring of $S$ which expressible as a directed union of Artinian subrings. On the other hand, if $\alpha$ is countable, then for each $i \geq 0$, 
let $F^{(i)}=\left\{\left\{x_{j}\right\}_{j=1}^{\infty} \in S: x_{i-1}=x_{i}=\cdots\right\}$ be a subring of $S$ isomorphic to $F^{i}$. According to [6, Proposition 5.1], $R=\bigcup_{i=1}^{\infty} F^{(i)}$ is a directed union of Artinian von Neumann regular subrings.

\section{References}

[1] D. D. Anderson, 'Some problems in commutative ring theory', in: Zero-dimensional commutative rings, (Knoxville, TN, 1994), Lecture Notes in Pure and Appl. Math. 171 (Dekker, New York, 1995) pp. 363-372.

[2] D. F. Anderson, A. Bouvier, D. E. Dobbs, M. Fontana and S. Kabbaj, 'On Jaffard domains', Exposition Math. 6 (1989), 145-175.

[3] M. F. Atiyah and I. G. Macdonald, Introduction to commutative algebra (Addison-Wesley, Reading, Massachusetts, 1969).

[4] R. Gilmer, 'Residue fields of zero-dimensional rings', in: Zero-dimensional commutative rings, (Knoxville. TN, 1994), Lecture Notes in Pure and Appl. Math. 171 (Dekker, New York, 1995) pp. 41-55.

[5] — 'Zero-dimensional subrings of commutative rings', in: Abelian groups and modules (Padova, 1994), Math. Appl. 343 (Kluwer, Dordrecht, 1995) pp. 209-219.

[6] R. Gilmer and W. Heinzer, 'Products of commutative rings and zero-dimensionality', Trans. Amer. Math. Soc. 331 (1992), 662-680.

[7] —-, 'Zero-dimensionality in commutative rings', Proc. Amer. Math. Soc. 115 (1992), 881-893.

[8] - 'Artinian subrings of a commutative ring', Trans. Amer. Math. Soc. 336 (1993), 295-310.

[9] _ - 'Imbeddability of a commutative ring in a finite-dimensional ring', Manuscripta Math. 84 (1994), 401-414.

[10] A. R. Magid. 'Direct limits of finite products of fields', in: Zero-dimensional commutative rings, (Knoxville, TN, 1994), Lecture Notes in Pure and Appl. Math. 171 (Dekker, New York, 1995) pp. 299-305.

[11] M. Nagata, Local rings (Interscience, New York, 1962).

\section{Department of Mathematics}

Faculty of Sciences Semlalia

P.O. Box 2390

Marrakech

Morocco

e-mail: ikarim@ucam.ac.ma 